\title{
ESTIMATIVA DA DETERIORAÇÃO DA MADEIRA DE ASSOALHO DE PRÉDIO HISTÓRICO POR MEIO DE ONDAS ULTRASSÔNICAS
}

\author{
Darci Alberto Gatto 1 , Margarete Regina Freitas Goncalves², Bruno Dufau Mattos ${ }^{3}$, \\ Leandro Calegari ${ }^{4}$, Diego Martins Stangerlin ${ }^{5}$
}

(recebido: 21 de fevereiro de 2011; aceito: 29 de junho de 2012)

\begin{abstract}
RESUMO: Esse trabalho apresentou como objetivo detectar o grau externo de deterioração da madeira de Araucaria angustifolia (Bert.) O. Kuntze utilizada na confecção de assoalho. Para isso, foram testados os métodos visuais, por meio de notas, e o método não destrutivo baseado na propagação de ondas ultrassônicas. Comparando-se a velocidade de propagação de ondas ultrassônicas na madeira sadia e na madeira atacada verificou-se que as mesmas diferenciavam entre si ao nível de 5\% de erro. Verificou-se também que o aumento na proporção de ataque na madeira apresentou-se inversamente proporcional à velocidade de propagação da onda. Uma vez que as amostras foram avaliadas apenas nas suas faces superficiais, fazem-se necessários estudos adicionais a fim de avaliar a influência dos defeitos internos das peças de madeira na velocidade ultrassônica.
\end{abstract}

Palavras-chave: Qualidade da madeira, método não destrutivo, onda de ultrassom.

\section{EVALUATION OF WOOD DECAY OF FLOOR OF HISTORIC BUILDING USING ULTRASONIC WAVES}

\begin{abstract}
This study aimed to detect the external degree of decay of Araucaria angustifolia (Bert.) $O$. Kuntze wood used in flooring . The visual method through grades and the non-destructive method based on the propagation of ultrasound waves were applied. The comparison between the propagation velocity of ultrasonic waves on clear wood and decayed wood showed statistical difference at the $5 \%$ level. Moreover, the proportion of decay wood was inversely proportional to the velocity of propagation. The samples were evaluated only on their superficial faces, therefore additional studies to assess the influence of the wood internal defects in the ultrasonic velocity are necessary.
\end{abstract}

Key words: Wood quality, non-destructive method, ultrasound waves.

\section{INTRODUÇÃO}

A avaliação não destrutiva da madeira tem tido aplicação crescente na avaliação da qualidade da madeira e apresenta vantagens em relação aos métodos convencionais, tais como possibilidade de avaliar a integridade estrutural de uma peça sem a extração de corpos de prova; maior rapidez para analisar uma grande população e versatilidade para se adequar a uma rotina padronizada numa linha de produção (OLIVEIRA; SALES, 2002). Diversas tecnologias são empregadas visando a esse objetivo, dentre as quais Ross (1999) cita o raios-X, análise química, propriedades vibracionais e transmissão de ondas sonoras.

A avaliação não destrutiva de madeiras com base nos princípios da propagação de ondas mecânicas, mais precisamente por meio da técnica de emissão de ultrassom, atualmente é um dos métodos mais aplicados e promissores, em razão da importância das informações que podem ser obtidas, da facilidade de operação do equipamento e do custo relativamente baixo na aquisição e operacionalização do mesmo.

\footnotetext{
${ }^{1}$ Engenheiro Florestal, Professor Doutor em Engenharia Florestal - Universidade Federal de Pelotas/UFPel - Centro de Engenharias Madeireira - Campus Capão do Leão s/nº - 96019-900 - Pelotas, RS, Brasil - darcigatto@ yahoo.com

${ }^{2}$ Engenheira Civil, Professora Doutora em Engenharia de Minas, Metalúrgica e de Materiais - Universidade Federal de Pelotas Faculdade de Arquitetura e Urbanismo - Departamento de Tecnologia da Construção - Rua Benjamin Constant, 1359, Centro Cx. P. 354 - 96010-900 - Pelotas, RS, Brasil - matgareterfg@ gmail.com

${ }^{3}$ Graduado em Engenharia Industrial Madeireira, Mestrando em Ciência e Engenharia de Materiais - Universidade Federal de Pelotas - Cx. P. 354 - 96010-900 - Pelotas, RS, Brasil - brunodufaumattos@yahoo.com.br

${ }^{4}$ Engenheiro Florestal, Professor Doutor em Ciência Florestal - Universidade Federal de Campina Grande - Centro de Saúde e Tecnologia Rural - Unidade Acadêmica de Engenharia Florestal - Bairro Santa Cecília - Patos, PB, Brasil - Cx. P. 64 - $58700-970$ calegari@cstr.ufcg.edu.br

${ }^{5}$ Engenheiro Florestal, Professor Doutor em Ciências Florestais - Universidade Federal de Mato Grosso/UFMT - Instituto de Ciências Agrárias e Ambientais - Avenida Alexandre Ferronato, 1200 - 78550-000 - Sinop, MT, Brasil diego_stangerlin@yahoo.com.br
} 
Diversas pesquisas demonstraram a viabilidade do emprego do método ultrassônico, por meio de correlações entre os módulos de elasticidade obtidos em ensaio destrutivos (compressão paralela e flexão estática) e em ensaios não-destrutivos. Os resultados apresentam correlações significativas com coeficientes de determinação $\left(\mathrm{R}^{2}\right)$ entre 0,57 e 0,89 (BARADIT et al., 1998; BARTHOLOMEU, 2001; MACHADO, 2000; NOGUEIRA, 2003; OLIVEIRA, 2001).

$\mathrm{Na}$ madeira, alguns fatores que influenciam a propagação de ondas ultrassônicas são: propriedades anatômicas, físicas (massa específica básica e aparente), morfológicas (tipos de lenhos e ângulo de grã), presença de defeitos (nós e rachaduras), geometria das amostras, condições do meio (temperatura e umidade relativa) e procedimentoutilizado para tomada das medidas (frequência e tipo de transdutor). Defeitos internos como rachaduras, colapsos, decomposição por microorganismos, bem como danos causados pelos cupins a estruturas de madeira podem acarretar riscos à vida e perdas econômicas. Dessa forma, a avaliação não destrutiva por meio de ondas ultrassônicas deve ser utilizada para avaliações periódicas relativas à qualidade da madeira, principalmente dos defeitos produzidos pela ação do tempo e dos agentes xilófagos.

Segundo Liñán et al. (2004), o método de propagação de ondas ultrassônicas é uma técnica eficaz para o diagnóstico de elementos estruturais de madeira em edifícios antigos. Esta técnica oferece uma grande ajuda na realização de um trabalho de restauro, por exemplo. De acordo com Halabe e Reynold (1999), a velocidade de propagação de ondas de choque, som ou ultrassom, depende diretamente da elasticidade do material. A madeira degradada perde a compactação do material celulósico tornando-se menos elástica que a madeira sã; dessa forma a velocidade da onda será menor na madeira degradada. Por outro lado, sabe-se que a onda mecânica propaga-se nos sólidos por agitação molecular, assim na madeira degradada a onda terá que contornar vazios, como os deixados por insetos e, dessa forma, levará mais tempo para ir do ponto de emissão ao ponto de recepção. Assim, uma baixa velocidade de transmissão poderá indicar a ocorrência de defeitos na madeira inspecionada.

Nesse contexto, esse trabalho apresentou como objetivo correlacionar o grau externo de deterioração da madeira de Araucaria angustifolia (Bert.) O. Kuntze, presente no assoalho de um prédio histórico do município de Pelotas - RS, com o método não destrutivo de propagação de ondas ultrassônicas.

Cerne, Lavras, v. 18, n. 4, p. 651-656, out./dez. 2012

\section{MATERIAL E MÉTODOS}

\subsection{Local de estudo}

O Casarão 8 (Figura 1) foi residência de Francisco Antunes Maciel, eminente personagem político do final do século XIX, o qual foi ministro da Justiça e Negócios Interiores no Governo de Getúlio Vargas.

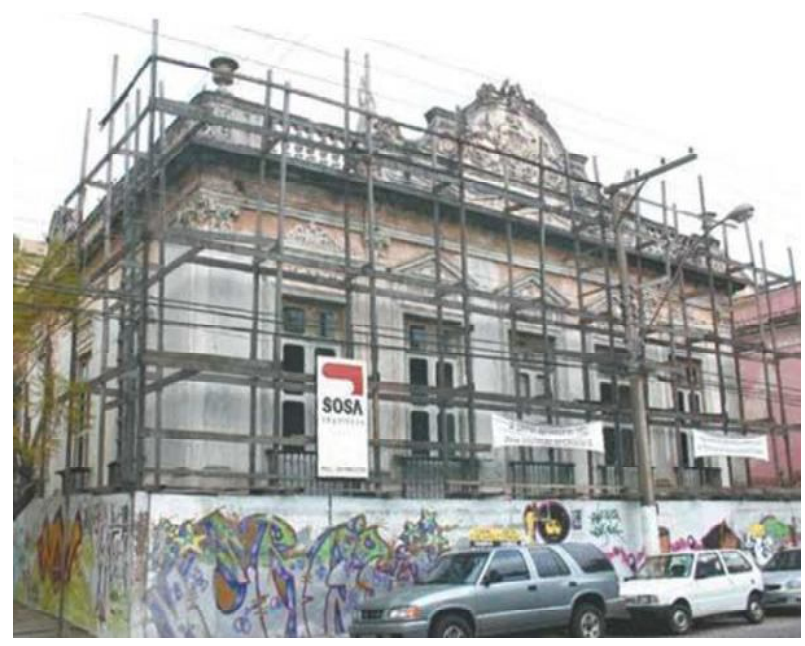

Figura 1 - Local de estudo: prédio histórico da cidade de Pelotas, RS.

Figure 1 - Object of study: historic building of Pelotas, Brazil.

Trata-se de uma das construções mais representativas do estilo eclético, o que ressalta sua importância arquitetônica. Nela é observada a presença de diversas técnicas de construção, tais como estuque de teto e parede e escariola de madeira.

O Casarão 8, prédio do Centro Histórico da cidade de Pelotas - RS, foi selecionado para o desenvolvimento de uma metodologia de classificação da sanidade da madeira utilizada no assoalho do referido prédio, como subsídio para futuras obras de restauro.

\subsection{Avaliação visual e ultrassônica}

O Casarão é constituído de 24 recintos, cujos assoalhos apresentavam danos produzidos basicamente por insetos e fungos. Em cada um dos recintos selecionaram-se ao acaso três locais de amostragem, onde em cada um deles realizou-se uma classificação visual, por meio de notas (Tabela 1), com posterior retirada de amostra de madeira para uma análise mais acurada. 
Tabela 1 - Nota de classificação em razão do dano causado pelos agentes xilófagos.

Table 1 - Score for classification according to the damage caused by xylophages agentes.

\begin{tabular}{clc}
\hline Nota & Avaliação do Dano Causado pelo Agente Xilófago & Recomendação \\
\hline 10 & Livre de qualquer patologia. & Restauro original \\
7 & $\begin{array}{l}\text { Ataque superficial, presença de poucos orifícios ou infecções (fungos de bolor ou } \\
\text { manchadores), sem qualquer comprometimento estrutural. }\end{array}$ & Tratamento superficial \\
5 & $\begin{array}{l}\text { Ataque moderado, presença de orifícios e infecções, fase inicial de apodrecimento, sem } \\
\text { comprometimento da peça. }\end{array}$ & Tratamento específico \\
3 & $\begin{array}{l}\text { Ataque intenso, presença de orifício, galerias, apodrecimento, comprometimento parcial do } \\
\text { uso da peça. }\end{array}$ & Substituição parcial \\
0 & Comprometimento da utilização da peça. & Substituição \\
\hline
\end{tabular}

Simultaneamente à análise visual, determinou-se a velocidade ultrassônica utilizando-se de um equipamento de emissão de ondas de ultrassom, acoplado a transdutores de faces planas de $2,5 \mathrm{~cm}$ de diâmetro útil e frequência de $45 \mathrm{kHz}$ da marca Procec modelo TICO.

A velocidade de deslocamento da onda ultrassônica, com os transdutores dispostos no modo semidireto (EVANGELISTA, 2002; NESVIJSKI, 2000) e com distância fixa de $20 \mathrm{~cm}$, foi determinada na direção longitudinal da madeira, uma vez que diversos pesquisadores (BARTHOLOMEU, 2001; COSTA, 2004; OLIVEIRA et al., 2005; PUCCINI, 2002) descreveram que essa direção apresenta o melhor ajuste operacional (Figura 2).

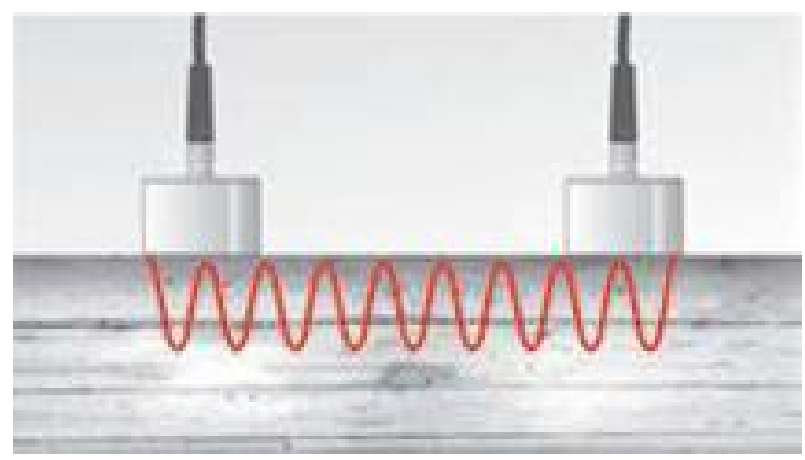

Figura 2 - Modo de disposição dos transdutores ultra-sônicos.

Figure 2-Method of disposition of ultrasonic transducers.

Antes de cada série de ensaios, o aparelho emissor de ondas de ultrassom foi calibrado conforme especificado no manual. Precedendo as calibrações e as determinações da velocidade ultrassônica no assoalho, foi aplicada uma fina camada de vaselina, servindo como acoplante às faces dos transdutores, evitando assim a presença de ar na interface.

\subsection{Determinação do nível de dano no assoalho}

Da parte do assoalho que apresentava danos foram tiradas fotografias com escala métrica e essas foram exportadas para um software CAD onde a porcentagem de dano observado no assoalho foi quantificada em relação à área total da imagem.

\section{RESULTADOS E DISCUSSÃO}

\subsection{Condições gerais do assoalho da residência}

Na Tabela 2, são mostrados os resultados de enquadramento quanto ao nível de dano do assoalho, analisado por meio da quantificação da área atingida, em relação ao método proposto por meio de notas qualitativas. Verifica-se que os recintos 8, 13 e 15 foram os mais afetados (cerca de $80 \%$ de sua área com Nota 0), todavia quase metade da área total analisada ainda encontrava-se em bom estado (Nota 10), e encontravam-se livres de agente xilófago.

Após a avaliação da degradação do assoalho do prédio, os autores sugerem, como boa opção para o material que recebeu as notas 0 e 3, a substituição com a mesma madeira. Por outro lado, para as notas 7 e 5 pode-se tratar inicialmente com inseticida e fungicida aplicado diretamente no local (orifício) ou expurgos, e quando ocorrer a presença de pontos apodrecidos, a escarificação torna-se uma boa solução. Paralelamente a isso é necessário fazer uma avaliação da umidade a fim de reduzi-la e evitar novos ataques. Outra alternativa é o uso de resinas para a recomposição de partes pequenas e/ou dos orifícios e galerias superficiais.

Cerne, Lavras, v. 18, n. 4, p. 651-656, out./dez. 2012 
Tabela 2 - Nível de dano causado por agentes xilófagos no assoalho construído com madeira de Araucaria angustifolia (Bert) O. Kuntze.

Table 2 - Damage level caused by xylophagous agents in floor built with wood of Araucaria angustifolia (Bert) O. Kuntze.

\begin{tabular}{|c|c|c|c|c|c|c|c|c|c|c|c|}
\hline \multirow{3}{*}{ Recinto } & \multirow{3}{*}{$\begin{array}{c}\text { Área de assoalho } \\
\left(\mathrm{m}^{2}\right)\end{array}$} & \multicolumn{10}{|c|}{ Notas } \\
\hline & & \multicolumn{2}{|c|}{0} & \multicolumn{2}{|c|}{3} & \multicolumn{2}{|c|}{5} & \multicolumn{2}{|c|}{7} & \multicolumn{2}{|c|}{10} \\
\hline & & $\%$ & $\mathrm{~m}^{2}$ & $\%$ & $\mathrm{~m}^{2}$ & $\%$ & $\mathrm{~m}^{2}$ & $\%$ & $\mathrm{~m}^{2}$ & $\%$ & $\mathrm{~m}^{2}$ \\
\hline 1 & 27,55 & 43,38 & 11,95 & 11,78 & 3,25 & 1,16 & 0,32 & 12,61 & 3,47 & 31,07 & 8,56 \\
\hline 2 & 51,77 & 2,62 & 1,36 & 3,51 & 1,82 & 8,37 & 4,33 & 25,78 & 13,35 & 59,72 & 30,92 \\
\hline 3 & 63,80 & 8,00 & 5,10 & 15,20 & 9,70 & 11,34 & 7,23 & 24,35 & 15,54 & 41,11 & 26,23 \\
\hline 4 & 46,80 & 2,18 & 1,02 & 2,41 & 1,13 & 8,60 & 4,02 & 24,92 & 11,66 & 61,89 & 28,96 \\
\hline 5 & 21,30 & 1,28 & 0,27 & 2,00 & 0,43 & 5,32 & 1,13 & 6,26 & 1,33 & 85,14 & 18,13 \\
\hline 6 & 17,80 & 0,98 & 0,17 & 0,00 & 0,00 & 3,28 & 0,58 & 8,17 & 1,45 & 87,57 & 15,59 \\
\hline 7 & 4,60 & 7,80 & 0,36 & 5,00 & 0,23 & 0,00 & 0,00 & 10,89 & 0,50 & 76,31 & 3,51 \\
\hline 8 & 19,00 & 80,00 & 15,20 & 17,43 & 3,31 & 1,38 & 0,26 & 1,19 & 0,23 & 0,00 & 0,00 \\
\hline 9 & 23,10 & 1,18 & 0,27 & 0,19 & 0,04 & 12,83 & 2,96 & 22,42 & 5,18 & 63,38 & 14,64 \\
\hline 10 & 21,50 & 40,75 & 8,76 & 11,90 & 2,56 & 5,00 & 1,08 & 4,18 & 0,90 & 38,17 & 8,21 \\
\hline 11 & 56,90 & 0,00 & 0,00 & 0,00 & 0,00 & 0,39 & 0,22 & 1,22 & 0,69 & 98,39 & 55,98 \\
\hline 12 & 7,30 & 21,00 & 1,53 & 8,02 & 0,59 & 2,98 & 0,22 & 16,11 & 1,18 & 51,89 & 3,79 \\
\hline 13 & 30,06 & 82,00 & 24,65 & 4,27 & 1,28 & 5,23 & 1,57 & 8,50 & 2,56 & 0,00 & 0,00 \\
\hline 14 & 18,20 & 20,71 & 3,77 & 11,37 & 2,07 & 4,13 & 0,75 & 21,17 & 3,85 & 42,62 & 7,76 \\
\hline 15 & 10,64 & 75,00 & 7,98 & 10,00 & 1,06 & 10,00 & 1,06 & 5,00 & 0,53 & 0,00 & 0,00 \\
\hline 16 & 10,93 & 40,00 & 4,37 & 4,00 & 0,44 & 5,00 & 0,55 & 50,00 & 5,47 & 1,00 & 0,11 \\
\hline 17 & 17,30 & 14,74 & 2,55 & 1,70 & 0,29 & 0,00 & 0,00 & 5,66 & 0,98 & 77,90 & 13,48 \\
\hline 18 & 36,30 & 5,31 & 1,93 & 11,00 & 3,99 & 3,90 & 1,42 & 15,00 & 5,45 & 64,79 & 23,52 \\
\hline 19 & 60,23 & 70,20 & 42,28 & 10,12 & 6,10 & 6,30 & 3,79 & 4,00 & 2,41 & 9,38 & 5,65 \\
\hline Total & 545,1 & 24,5 & 133,53 & 7,0 & 38,28 & 5,8 & 31,51 & 14,1 & 76,72 & 48,6 & 265,03 \\
\hline
\end{tabular}

\subsection{Velocidade de propagação das ondas ultrassônicas em} função do ataque dos agentes xilófagos

De acordo com a Tabela 3 , verifica-se que a perda de velocidade de propagação da onda pode ser utilizada como um indicativo do grau de deterioração da madeira. A diferença entre a velocidade de onda observada na madeira sã e a velocidade de onda da menor nota foi de $65,2 \%$, Shaji et al. (2000), usando como referência os valores para a velocidade de propagação de ondas obtidos em peças sã, consideram que uma perda maior que $30 \%$ na velocidade de onda nas peças atacadas em relação as sãs, exigirá a manutenção desse material. Por outro lado, Liñán e Hita (1995), estudando a madeira de resinosas, consideram que a perda de velocidade de apenas $10 \%$ em relação à madeira sã já seria uma referência de perda de resistência e requereria uma atuação naquela área.
Quando comparada a velocidade de propagação das ondas ultrassônicas das amostras de assoalho sadia (Nota 10) com as atacadas (Notas 7, 5, 3 e 0) por qualquer dos agentes xilófagos, observou-se diferença estatisticamente significativa ao nível de 5\% de probabilidade de erro, sendo que a madeira atacada apresentou velocidade de propagação menor.

Esse comportamento pode ser explicado em razão do efeito causado pelos agentes xilófagos à massa específica da madeira e, consequentemente, à continuidade do meio. Segundo Oliveira e Sales (2002), a massa específica da madeira é resultado da variação das características anatômicas de cada espécie, dessa forma não há uma relação clara entre a massa específica e a velocidade de propagação da onda na madeira, todavia essa propriedade é o parâmetro mais próximo (prático) para indicar a

\section{Cerne, Lavras, v. 18, n. 4, p. 651-656, out./dez. 2012}


Tabela 3 - Velocidade de onda em função da degradação do assoalho do prédio.

Table 3 - Speed of wave in function of the degradation of the floor of the building.

\begin{tabular}{cccccccc}
\hline Nota & N. de amostras & $\begin{array}{c}\text { Média } \\
\left({\left.\mathrm{m} . \mathrm{s}^{-1}\right)}^{*}\right.\end{array}$ & DP & CV (\%) & Mínimo & Máximo & Perda (\%) \\
\hline 10 & 30 & $4330,0 \mathrm{a}$ & 992,39 & 22,9 & 3040 & 5880 & 0 \\
7 & 11 & $3006,3 \mathrm{~b}$ & 1195,73 & 39,7 & 1700 & 5290 & 30,5 \\
5 & 11 & $2507,3 \mathrm{bc}$ & 755,65 & 30,1 & 1150 & 3550 & 42,1 \\
3 & 10 & $1752,6 \mathrm{~cd}$ & 411,55 & 23,4 & 1310 & 2470 & 59,5 \\
0 & 8 & $1507,5 \mathrm{~d}$ & 316,08 & 20,9 & 1040 & 2090 & 65,2 \\
\hline Total & 70 & 3144,8 & 1416,48 & 45,0 & 1040 & 5880 & - \\
\hline
\end{tabular}

Em que: $\mathrm{DP}=$ desvio padrão $\left(\mathrm{m} \cdot \mathrm{s}^{-1}\right), \mathrm{CV}=$ coeficiente de variação e Perda $(\%)$ - perda da velocidade de onda em relação à madeira sã. Não existe diferença estatisticamente significativa entre médias que compartilham uma mesma letra na coluna, de acordo com o Teste LSD de Fisher ao nível de 95,0\% de confiança.

continuidade do meio, que de fato influencia na velocidade de propagação da onda. Assim, mudanças drásticas nessa propriedade influenciam indiretamente na velocidade de onda.

Comparando-se o nível de dano (proporção de dano) causado pelos agentes xilófagos no assoalho (Tabela 2) com a velocidade de propagação da onda ultrassônica observa-se que esse dano é inversamente proporcional à velocidade ultrassônica (Figura 3).

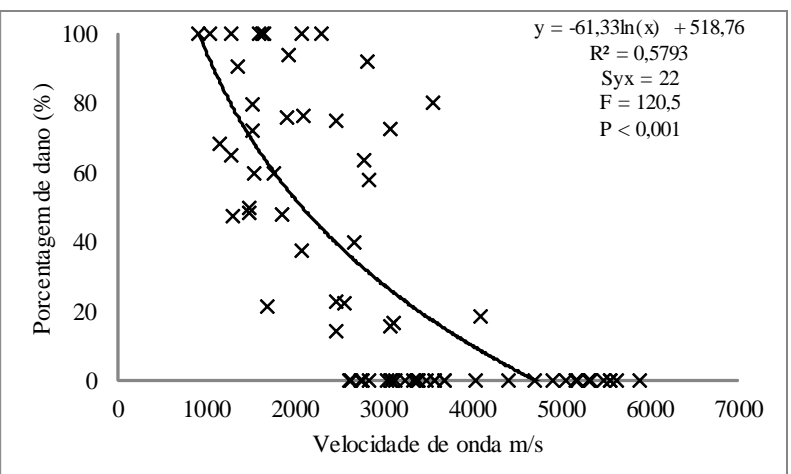

Figura 3 - Velocidade da onda ultrassônica em função da proporção de degradação da madeira.

Figure 3 - Ultrasonic wave velocity based on the amount of decayed wood.

Segundo Carrasco e Azevedo Júnior (2003), madeiras de maior massa específica apresentam maior teor de celulose e, consequentemente, um meio mais contínuo. Assim, a relação obtida entre a rigidez e a velocidade de onda no material dá-se por serem ambos dependentes da continuidade do meio. Portanto, o ataque de agentes xilófagos degrada os constituintes da madeira, provocando descontinuidades no meio, que afetam tanto a velocidade de propagação de ondas quanto a rigidez do material.

Reinprecht (2009), ao analisar vigas da Basílica de Egidius em Bardejov na Eslováquia, determinou que a menor velocidade de propagação de ondas ultrassônicas $\left(300-400 \mathrm{~m} . \mathrm{s}^{-1}\right)$ era principalmente nas extremidades das peças, onde estavam gravemente atacadas por podridão parda e possuíam visíveis galerias de insetos. Já, na parte central das peças, onde era menos evidente o ataque de fungos e insetos, a velocidade de ondas ultrassônicas ficou entre $700-1100 \mathrm{~m} \cdot \mathrm{s}^{-1}$.

O método de ultrassom, utilizado neste trabalho para avaliar a integridade de assoalhos de madeira, foi utilizado por Kandemir-Yucel et al. (2007), juntamente com termografias de infravermelho, para avaliar as condições de vigas de madeira em prédios históricos. Os dois estudos mostram que o método é sensível a degradação interna da madeira e se mostram como boa opção para obras de restauro, corroborando dados entre si.

\section{CONCLUSÕES}

Com os resultados obtidos no presente estudo foi possível concluir que:

- A velocidade de propagação de ondas mostrou comportamento inverso ao percentual de dano da madeira, caindo cerca de $70 \%$ da melhor situação (Nota 10) para a pior (Nota 0$)$;

- Para fins práticos o método ultrassonoro pode ser utilizado com o objetivo de inspecionar a sanidade de peças de assoalho nesse tipo de construção.

Cerne, Lavras, v. 18, n. 4, p. 651-656, out./dez. 2012 
No entanto, fazem-se necessários estudos adicionais a fim de avaliar a ocorrência de defeitos internos das peças de madeira, os quais não podem ser vistos superficialmente, mas que afetam consideravelmente a propagação das ondas ultrassônicas.

\section{REFERÊNCIAS}

BARADIT, E.; ROZAS, C.; VARGAS, G. Aplicación de ultrasonido en la evaluacion de madera de pinus radiata. Maderas: Ciencia y Tecnologia, Concepcion, v. 1, p. 27-33, 1998.

BARTHOLOMEU, A. Classificação de peças estruturais de madeira através do ultra-som. 2001. 105 p. Tese (Doutorado em Engenharia Agrícola) - Universidade Estadual de Campinas, Campinas, 2001.

CARRASCO, E. V. M.; AZEVEDO JÚNIOR, A. P. Avaliação não-destrutiva de propriedades mecânicas de madeiras através de ultra-som: fundamentos físicos e resultados experimentais. Cerne, Lavras, v. 9, n. 2, p. 178-191, 2003.

COSTA, O. A. L. da. Velocidade de propagação de ultrasom na madeira para diferentes condições de umidade. Campinas: UNICAMP, 2004. 47 p.

EVANGELISTA, A. C. J. Avaliação da resistência do concreto usando diferentes ensaios não-destrutivos. 2002. 219 p. Tese (Doutorado em Engenharia Civil) - Universidade Federal do Rio de Janeiro, Rio de Janeiro, 2002.

HALABE, U. B.; REYNOLD, F. Detection of flaws in structural members using spectral analysis of ultrasonic signals. Non-Destructive Testing, London, v. 15, n. 3/4, p. 215-236, 1999.

KANDEMIR-YUCEL, A.; TAVUKCUOGLU , A.; CANERSALTIK, E. N. In situ assessment of structural timber elements of a historic building by infrared thermography and ultrasonic velocity. Infrared Physics \& Technology, Exeter, v. 49, p. 243-248, 2007.

LIÑÁN, C. R.; HITA, P. R. Evaluación del estado de la madera em obras de rehabilitación mediante técnicas de ultrasonidos y obtención de parâmetros resistentes. Informes de la

Construcción, Granada, v. 47, n. 440, p. 5-22, 1995.

LIÑÁN, C. R.; HITA, P. R.; CÓZAR, J. C. G.; GÁLVEZ, F. P. Diagnóstico mediante técnicas de ultrasonidos del forjado de madera del refectorio del convento de santa clara en Carmona (Sevilla). Informes de la Construcción, Granada, v. 55, n. 490, p. 17-28, 2004.

MACHADO, J. M. R. S. S. Avaliação da variação das propriedades mecânicas de pinho bravo (Pinus pinaster Ait.) por meio de ultra-som. 2000. 265 p. Tese (Doutorado em Engenharia Florestal) - Universidade Técnica de Lisboa, Lisboa, 2000.

NESVIJSKI, E. G. On design of ultrasonic transducers and accuracy of velocity measurements. The e-Journal of Nondestructive Testing, Berlin, v. 5, n. 2, 2000.

NOGUEIRA, M. Determinação de módulos de elasticidade à compressão da madeira de Pinus taeda $\mathbf{L}$. com o uso de ultra-som. 2003. 149 p. Dissertação (Mestrado em Agronomia) - Universidade Estadual Paulista "Júlio de Mesquita Filho", Botucatu, 2003.

OLIVEIRA, F. G. R. de. Estudo de propriedades mecânicas de dicotiledôneas por meio de ensaios não destrutivos utilizando equipamento de ultra-som. 2001. 55 p. Dissertação (Mestrado em Ciência e Engenharia de Materiais) Universidade de São Paulo, São Carlos, 2001.

OLIVEIRA, F. G. R. de et al. Influência da seção transversal na velocidade ultra-sônica na madeira de Eucalyptus citriodora.

Cerne, Lavras, v. 11, n. 2, p. 197-203, 2005.

OLIVEIRA, F. G. R. de; SALES, A. Ultrasonic measurements in Brazilian hardwood. Materials Research, Pittsburgh, v. 5, n. 1 , p. $51-55,2002$.

PUCCINI, C. T. Avaliação de aspectos de qualidade da madeira utilizando o ultra-som. 2002. 139 p. Tese (Doutorado em Engenharia Agrícola) - Universidade Estadual de Campinas, Campinas, 2002.

REINPRECHT, L. Diagnostic of the degraded zones of fir beam situated in the st. Egidius' basilica in Bardejov. Forestry and Wood Technology, Berlin, v. 67, p. 201-207, 2009.

ROSS, R. J. Using sound to evaluate standing timber: review. Forest Products Journal, Madison, v. 1, n. 1, p. 43-44, 1999.

SHAJI, T.; SOMAYAJI, S.; MATHEWS, M. S. Ultrasonic pulse velocity technique for inspection and evaluation of timber. Journal of Materials in Civil Engineering, New York, v. 12, n. 2, p. 180-185, 2000.

Cerne, Lavras, v. 18, n. 4, p. 651-656, out./dez. 2012 